\title{
Next generation sequencing analysis reveals a relationship between rDNA unit diversity and locus number in Nicotiana diploids
}

Roman Matyášek ${ }^{1 \dagger}$, Simon Renny-Byfield ${ }^{2,3 \dagger}$, Jaroslav Fulneček ${ }^{1}$, Jiří Macas ${ }^{4}$, Marie-Angele Grandbastien ${ }^{5}$, Richard Nichols², Andrew Leitch² and Aleš Kovař́k ${ }^{*}$

\begin{abstract}
Background: Tandemly arranged nuclear ribosomal DNA (rDNA), encoding 18S, 5.85 and 265 ribosomal RNA (rRNA), exhibit concerted evolution, a pattern thought to result from the homogenisation of rDNA arrays. However rDNA homogeneity at the single nucleotide polymorphism (SNP) level has not been detailed in organisms with more than a few hundred copies of the rDNA unit. Here we study rDNA complexity in species with arrays consisting of thousands of units.

Methods: We examined homogeneity of genic (18S) and non-coding internally transcribed spacer (ITS1) regions of rDNA using Roche 454 and/or Illumina platforms in four angiosperm species, Nicotiana sylvestris, N. tomentosiformis, N. otophora and N. kawakamii. We compared the data with Southern blot hybridisation revealing the structure of intergenic spacer (IGS) sequences and with the number and distribution of rDNA loci.

Results and Conclusions: In all four species the intragenomic homogeneity of the 185 gene was high; a single ribotype makes up over $90 \%$ of the genes. However greater variation was observed in the ITS1 region, particularly in species with two or more rDNA loci, where $>55 \%$ of rDNA units were a single ribotype, with the second most abundant variant accounted for $>18 \%$ of units. IGS heterogeneity was high in all species. The increased number of ribotypes in ITS1 compared with 185 sequences may reflect rounds of incomplete homogenisation with strong selection for functional genic regions and relaxed selection on ITS1 variants. The relationship between the number of ITS1 ribotypes and the number of rDNA loci leads us to propose that rDNA evolution and complexity is influenced by locus number and/or amplification of orphaned rDNA units at new chromosomal locations.
\end{abstract}

Keywords: rRNA genes, Concerted evolution, Plants, Chromosomes, DNA repeats homogenisation

\section{Background}

In most eukaryotes, $5 \mathrm{~S}$ and $18-5.8-26 \mathrm{~S}$ nuclear ribosomal DNA (rDNA) units occur in tandem array at one or several loci. Each large rDNA unit (designated $45 \mathrm{~S}$ and $35 \mathrm{~S}$ for animals and plants, respectively) contains three different rRNA genes (18S, 5.8S and 26S), which are separated by two internal transcribed spacer sequences (ITS1 and ITS2). Each group is separated by the intergenic spacer (IGS, [1]). The genic regions are highly

\footnotetext{
* Correspondence: kovarik@ibp.cz

${ }^{\dagger}$ Equal contributors

'Institute of Biophysics, Academy of Sciences of the Czech Republic, v.v.i, Královopolská 135, Brno CZ-612 65, Czech Republic
}

Full list of author information is available at the end of the article conserved, whereas ITS divergence is sufficient to resolve species relationships within most genera. The IGS, which contains the transcription-start site and genetic and epigenetic features that influence the regulation of downstream genes, diverges more rapidly than the ITS.

Of particular interest to evolutionary biologists is the pattern of divergence of the whole rDNA array known as concerted evolution, in which the units of the rDNA array are very similar within a species but diverge between species. This pattern suggests that the arrays are subject to homogenisation - so that novel variants arising by mutation spread relatively rapidly along the array within any one species [2-4]. Computer modelling has suggested homogenisation would act to reduce mutational load and

\section{Biomed Central}


could therefore be favoured by selection $[5,6]$. An alternative model of multigene family evolution, perhaps most generally applicable to small multigene families, argues for birth and death of duplicate gene copies associated with selection, explaining relatively high diversity observed in gene families $[7,8]$. It has been suggested that this model might explain the unusually high levels of ITS and IGS polymorphisms in some species (up to $40 \%$ in some cases) [9-11].

In angiosperms, rDNA evolution has been challenging to study, since there are many copies of the large $35 \mathrm{~S}$ unit, amounting to thousands, or tens of thousands of units [12] distributed over one or several chromosomal loci [13]. High copy-number and sequence conservation of tandemly-arranged units make the analysis of the structure and organisation of the entire array difficult by classical cloning and sequencing approaches. Consequently, rDNA homogeneity at the single nucleotide polymorphism (SNP) level has not been comprehensively studied in any organism carrying more than a few hundred copies of the 35S rDNA unit. The advent of next generation sequencing (NGS) allows such an analysis for the first time $[14,15]$; in this study we therefore took advantage of NGS to explore rDNA homogeneity in four angiosperm species in the genus Nicotiana (Solanaceae).

Nicotiana is an ideal choice for the study of rDNA variation because we can take advantage of existing studies on the evolutionary processes operating across the genus. These studies at the cytogenetic [16-18], molecular [19,20] and genomic [21-23] levels have given insights into species relationships and patterns of species divergence. There are several phylogenetic studies examining patterns of sequence divergence across the genus [24-27], one of which used ITS sequences to infer species relationships [27]. These data revealed the presence of allopolyploids and complex patterns of interspecific hybridisation at the diploid level. Cytogenetic studies in Nicotiana have revealed that $35 \mathrm{~S}$ rDNA can occupy as many as five loci on different chromosomes [17,28,29] and in some species of section Tomentosae sub-regions of the rDNA array may also be dispersed [30]. Rapid amplification of novel 35S rDNA units has been observed in the fourth generation descendents of synthetic allopolyploids, artificially created to resemble natural $N$. tabacum [31]. Indeed the natural allotetraploid N. tabacum, thought to have formed $<200000$ years ago from relatives of modern $N$. sylvestris and $N$. tomentosiformis [32,33], shows signatures of rapid concerted evolution, where most of the progenitor $35 \mathrm{~S}$ rDNA units have been replaced by a variant that most closely resembles the $N$. tomentosiformis unit type $[34,35]$.

In this work, we addressed inter- and intragenomic homogeneity of rDNA arrays in four diploid Nicotiana species using two platforms of next generation sequencing (Roche 454 and Illumina) coupled with classical Sanger method. We sequenced amplicons covering equal portions of coding (18S gene) and non-coding (ITS1) regions (Figure 1). With these data, we compared the families and levels of divergence of units in both regions and interpreted the data in relation to the numbers of rDNA loci and levels of divergence in the intergenic spacer sequences (IGS). We found evidence for nearcomplete homogeneity of coding sequence irrespective of locus and copy-number. The non-coding region shows significantly higher divergence within those species that harbour multiple rDNA loci.

\section{Methods}

\section{Plant material}

The following accessions were used: i) Nicotiana sylvestris Speg. \& Comes ac. ITB626 originating from the Tobacco Institute, Imperial Tobacco Group, Bergerac, France. ii) Nicotiana tomentosiformis Goodsp. ac. NIC 479/84 (Institute of Plant Genetics and Crop Plant Research, Gatersleben, Germany). iii) Nicotiana otophora Griesebach ac. 406/76 (Royal Botanic Gardens, Kew, UK) and iv) N. kawakamii Y. Ohashi (voucher FN568429, Natural History Museum, London, UK).

\section{Preparation of sequencing amplicons by emulsion PCR}

Emulsion PCR was used in all steps in order to prevent formation of chimeric DNA during amplification. We first separated individual rDNA units in genomic DNA by HindIII restriction enzyme digestion, for which there is at least one restriction site distal to the $18 \mathrm{~S}-5.8 \mathrm{~S}$ region [34]. About $15 \mu \mathrm{g}$ of genomic DNAs from N. sylvestris, $N$. tomentosiformis, $N$. kawakamii and $N$. otophora

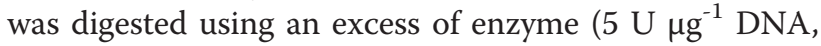
twice for $8 \mathrm{~h}$ ). Digested DNAs were precipitated with isopropanol, washed with $70 \%$ ethanol and re-dissolved in TE to concentrations of about 150-200 ng $\mathrm{Hl}^{-1}$.

For the emulsion PCR we essentially followed the protocol of Williams et al. [36]. Oil-surfactant mixture was prepared by thorough mixing of Span 80 (225 $\mu \mathrm{l}$; SIGMA, USA), Tween 80 (20 $\mu \mathrm{l}$; DIFCO, UK), Triton X100 (2.5 $\mu \mathrm{l}$; SIGMA, USA) and mineral oil (to $5 \mathrm{ml}$; SIGMA, USA) in a $15-\mathrm{ml}$ centrifuge tube at $25^{\circ} \mathrm{C}$. Four hundred $\mu \mathrm{l}$ of the oil-surfactant mixture was transferred into a CryoTube vial and stirred until use.

The composition of the aqueous phase $(230 \mu \mathrm{l})$ for the emulsion was as follow: 1x PfuUltra II reaction buffer, $10 \mathrm{~g}$ $\mathrm{l}^{-1} \mathrm{BSA}, 300 \mathrm{nM}$ each forward and reverse primer, $200 \mu \mathrm{M}$ each dNTP, $5 \mathrm{ng} \mathrm{ul}^{-1}$ of template DNA and $4.6 \mu \mathrm{l}$ of PfuUltra II Fussion HS DNA Polymerase (Stratagene, USA). The amount of genomic DNA was reduced to a minimum in order to maintain an excess of aqueous droplets over template molecules. The tripartite structure of 
primers was as follows: 454 sequencing adaptors (italics), variable hexanucleotide TAG sequence (normal letter) and a gene specific 3' end (bold). Primer A (5'-CGTATCG CCTCCCTCGCGCCATCAGTCGTATCTACACTGATGT ATTCAACGAG-3') contained the conserved 18S gene; Primer B (5'-CTATGCGCCTTGCCAGCCCGCTCAGTCG TATCCGTTGCCGAGAGTCGTTT-3') was derived from the $5.8 \mathrm{~S}$ gene (Figure 1). The gene-specific parts are identical to $18 \mathrm{~S}-\mathrm{FOR}$ and $5.8 \mathrm{~S}-\mathrm{REV} 2$ primers used in previous projects [37] and do not seem to discriminate between individual alleles. Two hundred $\mu \mathrm{l}$ of the aqueous phase was added in a drop-wise manner to the $400 \mu \mathrm{l}$ of stirred oilsurfactant mixture for over a period of 1.5-2.0 min. Stirring was continued for an additional $5 \mathrm{~min}$ and then the whitish emulsion was divided into $50 \mu \mathrm{l}$ aliquots. Approximately $20 \mu \mathrm{l}$ of aqueous phase was used as a control. The samples were overlaid with a drop of mineral oil and subjected to temperature-cycling: initial denaturation at $94^{\circ} \mathrm{C}$ for $3 \mathrm{~min}$ was followed by 25 cycles of $94^{\circ} \mathrm{C}$ for $30 \mathrm{~s}, 57^{\circ} \mathrm{C}$ for $30 \mathrm{~s}$ and $72^{\circ} \mathrm{C}$ for $40 \mathrm{~s}$. The emulsified PCR reactions were pooled into a $1.5 \mathrm{ml}$ microcentrifuge tube and centrifuged at $13,000 \mathrm{~g}$ for $5 \mathrm{~min}$ at room temperature. The upper (oil) phase was removed. In order to break the emulsion, 2-3 extractions with water-saturated diethyl ether were performed (approximately $1 \mathrm{ml}$ of ether was added, vortexed and the upper phase removed). Residual ether was removed by incubation $15 \mathrm{~min}$ at $42^{\circ} \mathrm{C}$ or by a centrifugation under vacuum for $5 \mathrm{~min}$ at room temperature. The PCR products were checked by agarose gel electrophoresis and cleaned using a PCR purification kit (Macherey-Nagel, Germany). The binding buffer was adjusted to remove all unincorporated primers according to the manufacturer's recommendation.

\section{Amplicon sequencing}

Samples containing 100-200 ng of each tagged PCR product were pooled and sent to EuroFins Operon MWG (Germany) for 454 sequencing. The pooled sample was sequenced from Primer A- and Primer B sides using a Roche GS FLX apparatus and Titanium series chemistry. Average length of reads was 350 to 450 base pairs and these reads are available at the NCBI Sequence Read Archive under the accession number SRA051350.

Amplicon variance analysis was carried out at Eurofins MWG Operon (Germany) and included Mira coverage mapping and syntheny plots. The SNPs were detected in the 454SNP/454HCDiff.files retrieved from the GsMapper. The criteria for clustering were as follows: All alignments were performed using the Smith-Waterman algorithm with default parameters with an identity threshold of e-100 and a maximum of 10 mutations per read. The reference sequences used for mapping were obtained from direct Sanger sequencing of $18 \mathrm{~S}-5.8 \mathrm{~S}$ PCR products. In general, half of the reads were successfully mapped to the reference sequences. The unmapped reads contained mostly shorter sequences or unrelated and highly variable sequences that were excluded from further analysis. The lengths of reference sequences were as follows: 313 bp of the $18 \mathrm{~S}$ (all species), $240 \pm 2 \mathrm{bp}$ of the ITS1. The $313 \mathrm{bp}$ of $18 \mathrm{~S}$ gene is delimited by positions $1689-1801$ of the $18 \mathrm{~S}$ gene from tomato (X51576.1); positions of ITS1 were according to the sequences deposited in GenBank (AJ492423.1, AJ492450.1, AJ492455.1, AJ492445.1, [27]). The sequences from major ( $>5 \%$ of total reads) clusters (equivalent to gene families) were imported to the BIOEDIT sequence editor [38] and checked by eye.

\section{Illumina genomic DNA sequencing}

Sequence reads were obtained from Illumina sequencing of $N$. sylvestris (ac. ITB626) and $N$. tomentosiformis (ac. NIC 479/84) genomic DNA using the Illumina Genome Analyzer xII at the Genome Centre Queen Mary University of London, as described in Renny-Byfield et al. [23]. A random sample between $47-61 \%$ of the genome was sequenced for each species. Sequence reads are available at the SRA under the study accession number SRA045794.1. Illumina reads were mapped to ITS reference sequences and SNP and DIP (insertion/deletion) analysis was carried out using GLC Genomics Workbench with the following parameters/ requirements: window length of $11 \mathrm{bp}$, maximum of 2 gaps, a minimum coverage of 4 , variants should occur at a minimum frequency of 0.01 , with a maximum of 1000 variants expected.

\section{Analysis of clones}

ITS sequences were obtained by PCR amplification of genomic DNA. In a $25 \mu \mathrm{l}$ reaction we used $0.1-1$ ng genomic DNA as template, $500 \mathrm{nM}$ each primer, $200 \mu \mathrm{M}$ each dNTP, and 0.4 units of DyNAzyme II DNA polymerase (Finnzymes, Espoo, Finland). Cycling conditions were as follows: an initial denaturation step of $94^{\circ} \mathrm{C}$ for $180 \mathrm{~s}$ followed by $15-35$ cycles of $94^{\circ} \mathrm{C}$ for $20 \mathrm{~s}, 57^{\circ} \mathrm{C}$ for $30 \mathrm{~s}$ and $72^{\circ} \mathrm{C}$ for $30 \mathrm{~s}$ with $18 \mathrm{~S}$-FOR (5'-GCGCTACACTGATG TATTCAACGAG-3') and 5.8S-REV (5'-CGCAACTTGCG TTCAAAGACTCGA-3') primers [37]. The products were purified using a PCR purification kit (Macherey-Nagel, Germany) and cloned into a dT vector (pDrive, Qiagen, Germany). Sequencing was carried out by dideoxy chaintermination at the Eurofins MWG Operon (Germany) using the SP6 and T7 primers. We considered the possibility of differential amplification of GC-rich and GC-poor templates $[39,40]$ and included 5\% dimethyl sulfoxide in the PCR reaction and found this had no effect on the proportion of amplified gene families.

\section{Southern blot hybridisation}

DNA was extracted from fresh young leaves according to Kovarik et al. [41], digested with EcoRV restriction 
endonuclease ( $5 \mathrm{U} \mathrm{\mu g}^{-1} \mathrm{DNA}$, twice for $6 \mathrm{~h}$ ), fractionated by gel electrophoresis and transferred to Hybond XL membranes (GE-Healthcare, UK) using alkaline capillary transfer. Membranes were hybridised with 32P-labelled DNA probe (DecaLabel DNA Labeling Kit, MBI Fermentas). Southern blot hybridisation was carried out in a $0.25 \mathrm{M}$ sodium phosphate buffer ( $\mathrm{pH}$ 7.0) supplemented with $7 \%(\mathrm{w} / \mathrm{v})$ sodium dodecyl sulphate (SDS) at $65^{\circ} \mathrm{C}$. Membranes were washed with $2 \mathrm{x}$ SSC, $0.1 \%$ SDS (twice for $5 \mathrm{~min}$ ) and then with $0.2 \mathrm{x}$ SSC and $0.1 \%$ SDS (twice for $15 \mathrm{~min}$ at $65^{\circ} \mathrm{C}$ ). The membranes were exposed to a Storage Phosphor Screen, scanned (Storm, GE-Healthcare, USA) and the signal was quantified using Image Quant (GE-Healthcare, USA). The DNA probe was an insert of the clone carrying the $18 \mathrm{~S}$ gene sequence from tomato (GeneBank number X51576).

\section{Data access}

All reads are available at the NCBI Sequence Read Archive (SRA) under the accession numbers SRA051350 and SRA045794.1. The cluster of reads used for mutation analysis can be accessed from: http://www.ibp.cz/local/ data/matyasek/.

\section{Results}

We used emulsion PCR (for primer sites see Figure 1) to analyse the diversity of $18 \mathrm{~S}$ and ITS1 regions of rDNA in four Nicotiana species, $N$. tomentosiformis, $N$ sylvestris, $N$. otophora and $N$. kawakamii. PCR amplicons were sequenced using Roche 454 pyrosequencing and the resulting reads were mapped to reference sequences obtained by direct Sanger sequencing of PCR products (Table 1). The aligned sequences were sorted into clusters, where each cluster contained one or more reads with unique sequence (Additional files 1,2 ). We analysed the nature of SNPs that define clusters and found $\mathrm{C} \rightarrow \mathrm{T} / \mathrm{G} \rightarrow \mathrm{A}$ transitions were the most frequent polymorphisms in both $18 \mathrm{~S}$ and ITS1 sequences, likely to be a consequence of high frequency mutations caused by deamination of methyl cytosine to thymine (Additional file 3 ). The percentage contribution each cluster makes to the rDNA complement is shown in Additional file 4. In each species, there was a single cluster of $18 \mathrm{~S}$ sequences that comprised more than $70 \%$ of the reads, while the remaining clusters were read-poor (less than $1 \%$ of the total reads). ITS1 sequences were more diverse, with two to six clusters exceeding $3 \%$ of reads depending on the species. However, sequencing using Illumina technology indicated that clusters defined by indels were likely to be artefacts (see later). Therefore we disregarded indels, and redistributed the reads to other clusters based on nucleotide polymorphisms only. Furthermore, singletons (clusters with one read) may also be sequencing and/or PCR artefacts, and so these were also excluded from further analysis. This markedly reduced the number of clusters (from $>300$ to less than 60) in each species (Figure 2). The majority (>90\%) of $18 \mathrm{~S}$ reads in each species occurred in one cluster, while the remaining clusters were

\section{A}

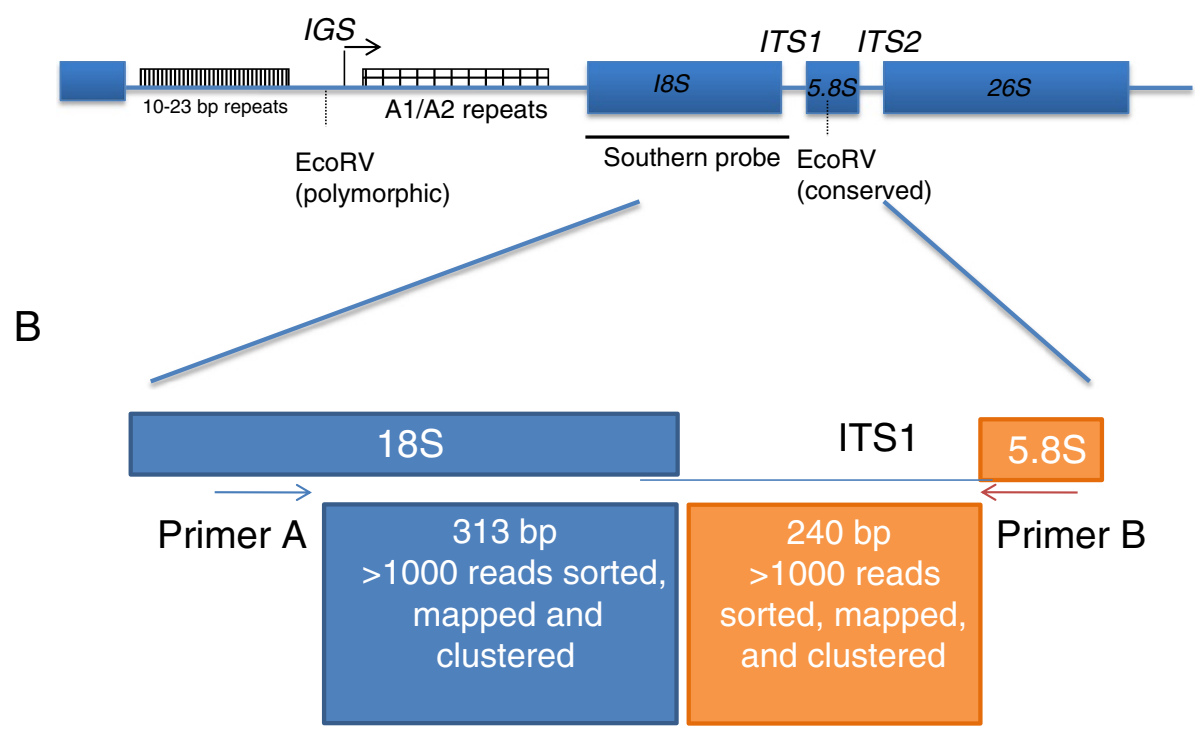

Figure 1 (A) Structure of 35S rDNA unit in Nicotiana (redrawn from [34]). The transcription start site is arrowed. IGS = intergenic spacer, ITS = internal transcribed spacer (B) PCR primer binding sites to the $18 \mathrm{~S}$ and $5.8 \mathrm{~S}$ rDNA unit and 454 sequencing reads generated. The total amplicon length was $650 \mathrm{bp}$. 
Table 1 Summary of 454 sequencing data

\begin{tabular}{|c|c|c|c|c|c|c|c|c|}
\hline \multirow[t]{2}{*}{ Species } & \multirow[t]{2}{*}{ Number of loci1 } & \multirow[t]{2}{*}{ Copies/1C2 } & \multicolumn{2}{|c|}{ Reads mapped } & \multicolumn{2}{|c|}{ Coverage3 } & \multicolumn{2}{|c|}{ Number of clusters } \\
\hline & & & $18 S$ & ITS1 & $18 \mathrm{~S}$ & ITS1 & $18 \mathrm{~S}$ & ITS1 \\
\hline N. tomentosiformis & 1 & 1400 & 1190 & 1592 & 0.9 & 1.1 & 204 & 190 \\
\hline N. sylvestris & 3 & 5100 & 2657 & 3882 & 0.5 & 0.8 & 339 & 483 \\
\hline N. otophora & 2 & 1500 & 1469 & 2308 & 1.0 & 1.5 & 105 & 303 \\
\hline N. kawakamii & 3+dispersed & 2200 & 998 & 1093 & 0.5 & 0.5 & 138 & 213 \\
\hline
\end{tabular}

${ }^{1}$ See Figure 6 and Plant rDNA database - www.plantrdnadatabase.com [42].

${ }^{2}$ The gene copies were initially determined by slot blot hybridisation [43] and later adjusted by calculation from the NGS data [22] using a http://w3lamc.umbr.cas. cz/profrep/ server. For N. kawakamii and N. otophora the copies were determined based on Southern blot signals (Figure 5).

${ }^{3}$ Mean read-depth: the number of aligned reads divided by rDNA copy number.

read-poor $(<1.5 \%$ of reads). Thus for each species $18 \mathrm{~S}$ rDNA homogeneity is high, indicating that these regions are largely formed by one ribotype. In the case of ITS1 sequences, there are differences among species: in $N$. tomentosiformis there is a single abundant ITS1 cluster (ribotype); however, in N. sylvestris, $N$. kawakamii and $N$. otophora there are multiple read-rich ITS1 clusters (2-3 of which exceed 5\% of the total number of reads).

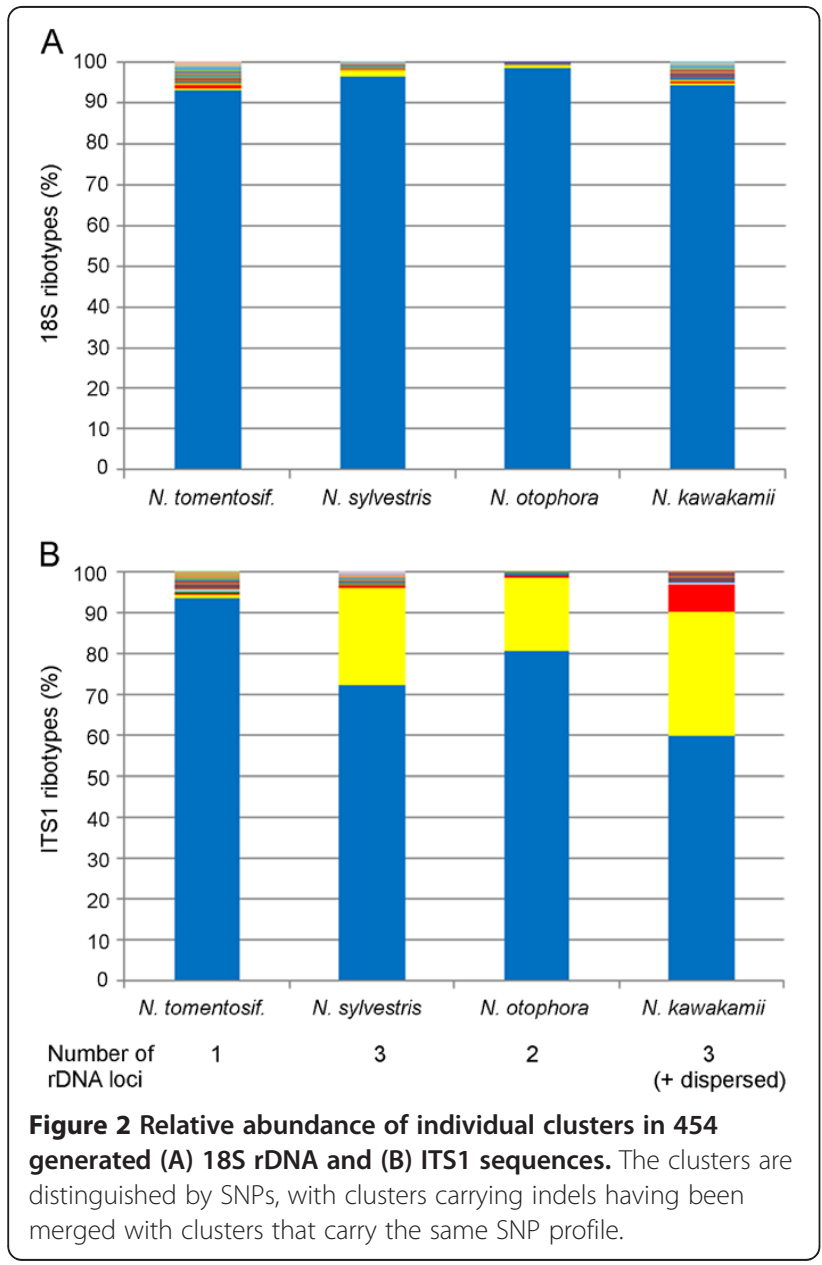

\section{Illumina sequencing}

Illumina reads from $N$. sylvestris and $N$. tomentosiformis were mapped to reference ITS1 sequences obtained from Sanger sequencing of PCR products from both species using the "map reads to reference" function of CLC Genomics Workbench. The read-depth for each nucleotide position along the ITS region varied from 239 to 750. Subsequently, we analysed mutational variation using SNP and DIP detection algorithms in the CLC Genomics Workbench (Additional files 5, 6). Out of 240 nucleotide positions, there are 140 (58\%) and 154 (64\%) polymorphic sites in N. tomentosiformis and N. sylvestris, respectively. At variant positions, deviation from the most common nucleotide was rare, in most cases and did not exceed $1 \%$ of the reads, as previously observed in 454 sequence data. Substitution polymorphisms occurring at $>1 \%$ frequency were counted and are presented graphically in Figure 3. Of the sites that are polymorphic, the frequency of the commonest SNP allele was high, with polymorphisms influencing only a minor fraction of sequences (minor

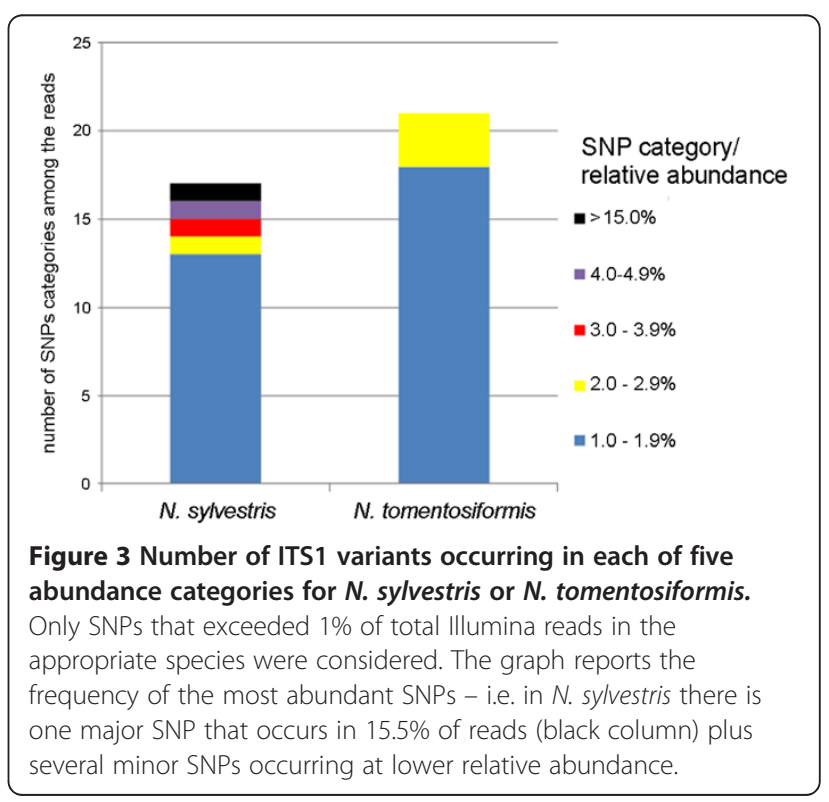


SNPs). Of these minor SNPs, the commonest occurred in only $2.4 \%$ of reads in $N$. tomentosiformis and $15.5 \%$ of reads in N. sylvestris (Figure 3). The position of predominant polymorphic sites matched those identified by 454 amplicon sequencing.

\section{Comparing data between sequencing methods}

To validate the results we compared frequencies of SNPs obtained with different sequencing methods across $N$. sylvestris ITS1 sequences (Additional file 7). We cloned 18S-ITS1 PCR products from N. sylvestris and sequenced (15 clones) by Sanger method. As expected, we did not observe any variation in the $18 \mathrm{~S}$ region, confirming high levels of homogeneity. However, three clones $(20 \%)$ contained a $\mathrm{C} \rightarrow \mathrm{T}$ substitution at position 57 in ITS1. These clones were identical to the 454 reads in the second most abundant cluster (Figure 2, yellow field). Likewise SNP analysis of Illumina datasets revealed the same substitution, occurring at a frequency of $15.5 \%$ (Figure 3). Thus all three methods are broadly comparable.

Among the $3882 N$. sylvestris ITS sequences derived from 454 data, there were 680 reads with indels and 800 reads harbouring substitutions (1.2 substitution/indel ratio). The ratio was significantly higher (18.1 substitution/ indel ratio) among the Illumina reads indicating that most indels in the 454 data are likely to be artefacts. Indeed, visual inspection of sequences showed that most indels were actually located in homopolymeric tracts, where pyrosequencing is known to introduce errors [44]. Illumina seems to be less prone to indel artefacts, although even in this case, one abundant indel variant (15\% reads) could not be traced among the 454 reads or Sanger sequences. We recommend caution when interpreting indel polymorphisms from such NGS data, and suggest using a combination of approaches.

\section{Phylogenetic relationships between the ITS1 families}

To determine phylogenetic relationships between ITS ribotypes, we extracted a single ITS ribotype from $N$. tomentosiformis, two from $N$. sylvestris and $N$. otophora, and three from $N$. kawakamii, based on a minimum 5\% representation in the corresponding species. The sequences were aligned and phylogenetic trees constructed. Maximum Likelihood (ML) and Neighbour joining trees had similar topologies. The ML tree (Figure 4) revealed a relatively large phylogenetic distance between ITS1 ribotype in $N$. sylvestris and ribotypes in the three other species. This is expected since N. sylvestris is placed within the section Sylvestres while the rest of species belong to section Tomentosae [45]. Amongst sequences from section Tomentosae, the two ITS1 families of $N$. otophora form a distinct clade, whilst those from $N$. kawakamii fall into two groups. Next,

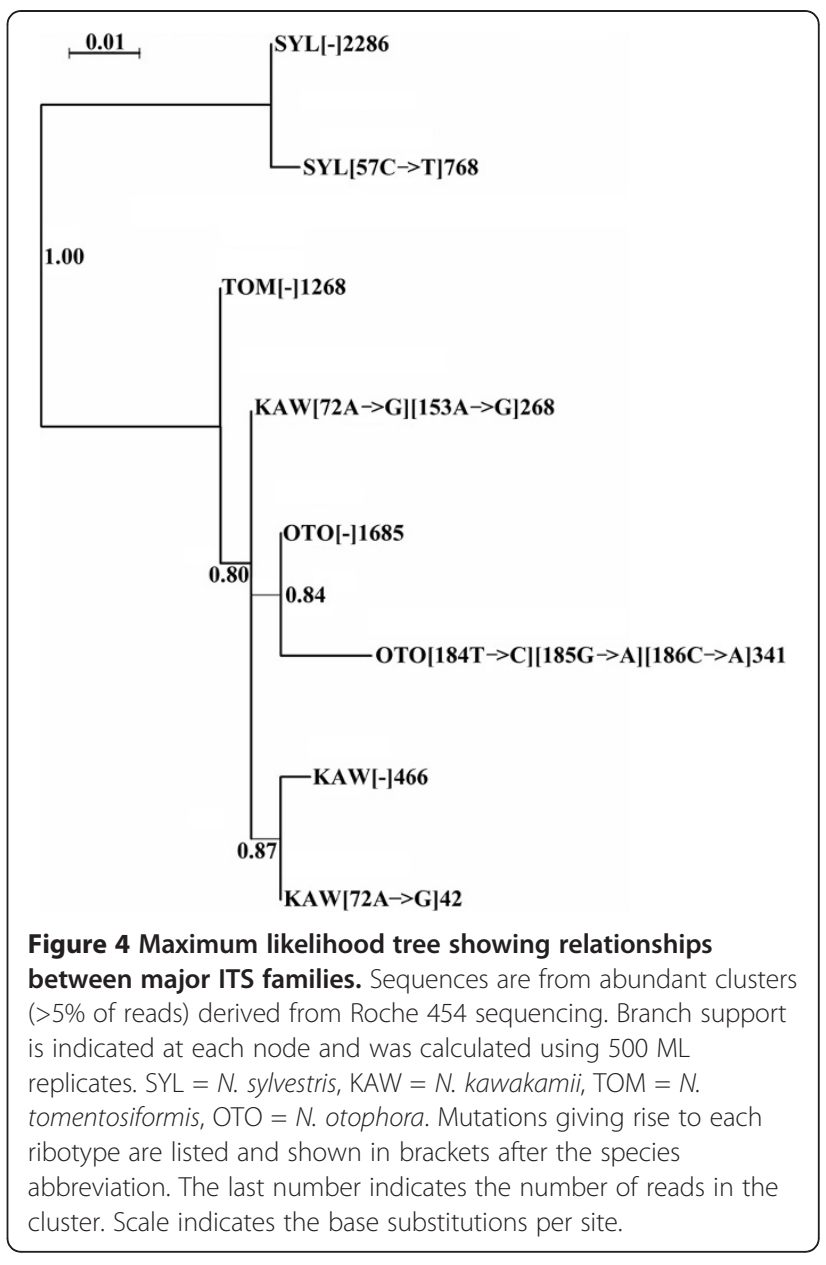

using BLAST searches we determined if ITS variants in one species were present in low copy number in other species. However, abundant ribotypes appeared to be unique for each species and did not appear in other species, even among low-copy clusters.

\section{Southern blot hybridisation}

The intergenic spacer (IGS, Figure 1) is internally repetitive making the mutation analysis of this region difficult. The individual sub-repeats within the IGS showed differential coverage in the 454 NGS data sets (Additional file 8). We therefore carried out Southern blot hybridisation to determine intragenomic homogeneity of IGS sequences. Genomic DNAs were digested with EcoRV restriction enzyme to release part of the IGS and a complete $18 \mathrm{~S}$ gene. The restriction fragments were probed with $18 \mathrm{~S}$ rDNA (Figure 5). It is evident that besides a major single hybridisation band there were additional bands with a weaker hybridisation signal. The fainter fragments formed a ladder of bands spaced at $135 \mathrm{bp}$ intervals, indicating point mutations and/or variable number of A1/A2 subrepeats located downstream of the promoter $[30,34]$. Thus, as with 


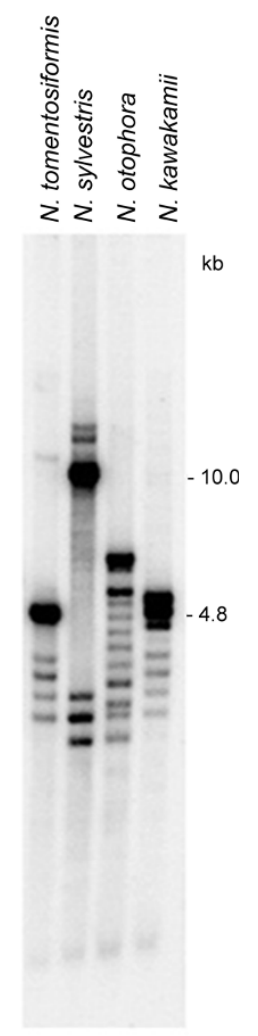

Figure 5 Southern blot hybridisation shows the presence of multiple IGS variants in the Nicotiana genomes. There were at least 14 EcoRV bands in N. otophora.

the IGS displays significant levels of intragenomic heterogeneity. Analysis of sequence coverage revealed substantial differences in the lengths and homogeneity of IGS subrepeats between $N$. tomentosiformis and $N$. sylvestris (Additional file 8). The upstream and downstream repeated elements have differentially amplified between both species [34].

\section{Discussion}

To study concerted evolution of rDNA arrays during the divergence of diploid Nicotiana species, we analysed SNPs in the 18S (coding) and ITS1 (non-coding) regions using Roche 454 and Illumina technology. The coverage of both 18S (3' end) and ITS1 regions of rDNA was close to 1 , meaning that most rDNA variants are likely to be represented in our dataset. We show that $18 \mathrm{~S}$ sequences are more homogenous than ITS1; a pattern that could arise from incomplete rounds of homogenisation and stronger purifying selection acting on the $18 \mathrm{~S}$ genic region. By analysing genomic and cytogenetic data, we find evidence that, in Nicotiana, the separation of rDNA arrays between chromosomes influences patterns of rDNA homogenisation. We explore these ideas in more detail below.

\section{Homogeneity in coding and non-coding regions of the rDNA unit}

Nicotiana tomentosiformis has only one read rich-cluster (defined as a cluster comprising more than 5\% of the reads) for both $18 \mathrm{~S}$ and ITS1 sequences (Figure 2). These data demonstrate near complete homogenisation of both $18 \mathrm{~S}$ and ITS1 sequences to a single ribotype. For the other diploid species $N$. sylvestris, $N$. kawakamii and $N$. otophora there was also a single read rich $18 \mathrm{~S}$ rDNA cluster, but there were two or three read rich ITS1 clusters that differed by 1-3 substitutions per 240 bp (0.4-1.3\% divergence; Figure 2). Thus, within each of these species the $18 \mathrm{~S}$ gene is more homogenous than the ITS1. The average number of major ITS1 variants in Nicotiana is similar to the average number (2-3) of ITS2 ribotypes recently determined for a wide range of plant species by pyrosequencing [14] corroborating the hypothesis that amplification and fixation of rDNA variants could be larger in plants than in yeasts [46] and Drosophila [47]. Consistent with previous studies $[48,49]$, for IGS sequences there is even greater complexity in the Nicotiana species analysed (Figure 5) probably due to its subrepeated nature ([34] and Additional file 8). Increasing genetic variation, $18 \mathrm{~S}$ < ITS1 < IGS likely reflect reducing strengths of selection. Probably the IGS homogenises by similar mechanisms (e.g. unequal cross over) as the rest of the unit since the first and last A1/A2-subrepeats (Figure 1) are more variable than those of the central region [50], consistent with distance-dependent models of repeat evolution [51,52].

\section{Most rDNA ribotypes have not survived species divergence in Nicotiana}

Analyses of the occurrence and distribution of repetitive DNA sequences [17,53] and phylogenetic analyses of plastid (trnL-intron, trnL-F, trnS-G, ndhF, matK; [54]) and glutamine synthase [25] sequences indicate that $N$. tomentosiformis is recently derived within the section. Fluorescence in situ hybridisation studies indicate that the ancestral organisation for the section is most likely to have been two $35 \mathrm{~S}$ rDNA loci, located on chromosomes 3 and 4 (Figure 6, the homeology is supported by cytogenetic observations of satellite and endogenous virus repeats $[17,55])$. If so, there has been a gain in locus number in the lineage leading to N. kawakamii and loss of a locus in the lineage leading to N. tomentosiformis [17]. Nicotiana sylvestris occurs in the distantly related section Sylvestres and has three 35S rDNA loci, the homeologous relationship to loci with section Tomentosae is currently unknown. Despite the more derived position of $N$. tomentosiformis in Tomentosae, the one major ribotype identified is more similar to the $N$. sylvestris ribotype than others in section Tomentosae, indicative of array homogenisation maintaining an ancestral-like unit. For N. otophora and N. sylvestris, 
the ribotypes fall into species-specific clades. For N. kawakamii, two copy types form a clade and the third copy an independent clade (Figure 4), revealing the amplification of two distinct variants in this species. It is unlikely that these data are explained by incomplete lineage sorting $[10,56,57]$ of mixed rDNA arrays, since the 'major' ITS ribotypes in each species was not found in any other species. Indeed, there is evidence for the rapid establishment of ribotypes in other species: for example in the genus Hordeum, it is estimated that complete ITS replacement occurs within $\sim 3.5$ myrs [58] and deep sequencing of Arabidopsis thaliana show that ITS variants are differentially amplified between ecotypes [59]. Furthermore, in Nicotiana polyploids, we have estimated that there is near complete replacement of rDNA repeats in less than one million years
$[22,35]$. Thus the rate of ribotype homogenisation appears to exceed the rate of speciation.

\section{Chromosomal locus separation as a driver of ITS complexity}

Large scale analysis of rDNA repeats in yeasts [46] and Drosophila [47] has also revealed differences among rDNA units of the same species. However, unlike the pattern we report here, intragenomic homogeneity is similar in coding and non-coding regions of each species, although slightly higher variation was observed in Drosophila, where rDNA sequences are located at several chromosomal loci. In explaining the level of variation, it is suggestive that the Rosaceae [60,61] and Cactaceae [9], in which large numbers of ITS variants were observed, also have a high $(>5)$ number of rDNA

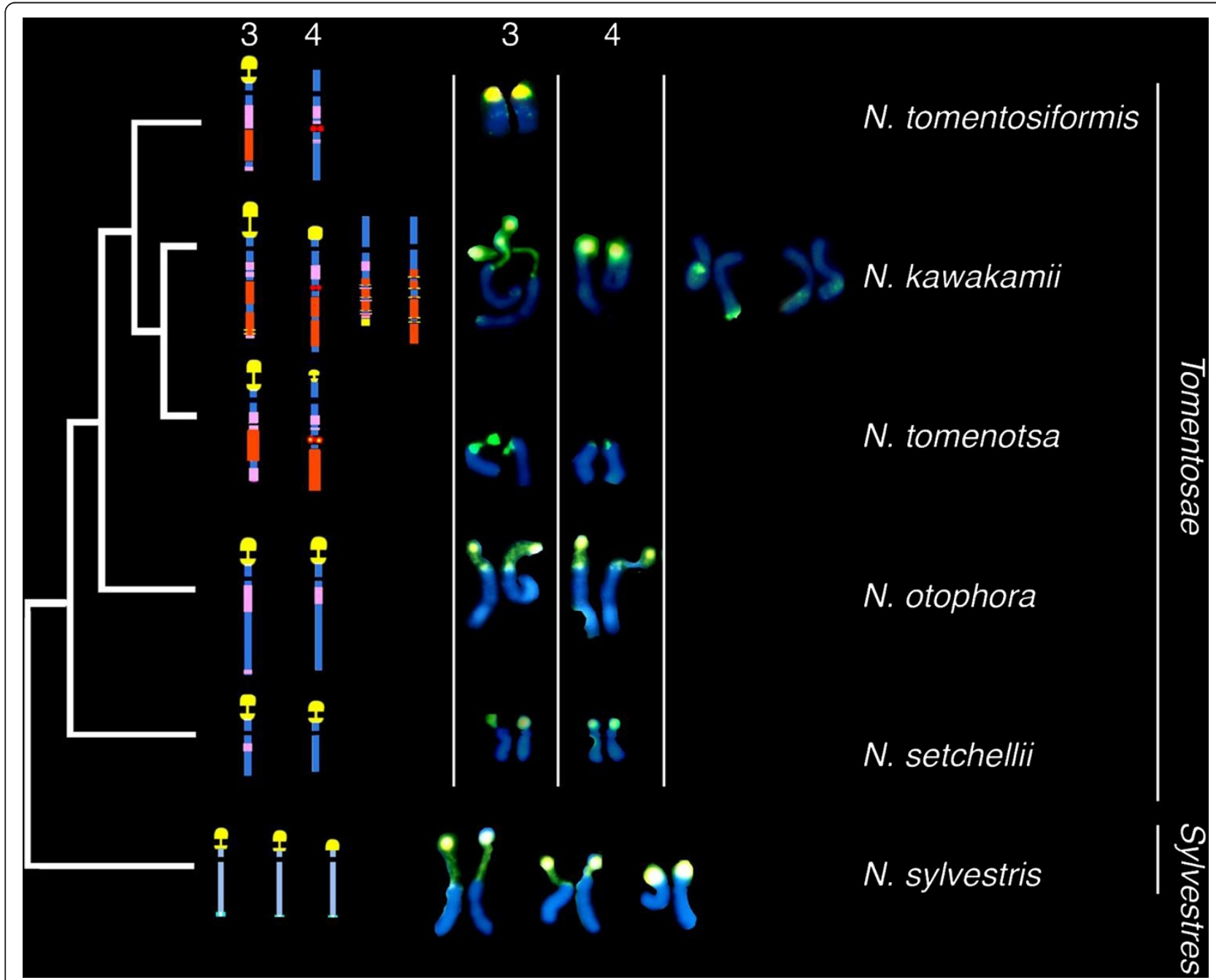

Figure 6 Phylogenetic relationships between species in section Tomentosae and $N$. sylvestris as determined by the distribution of repetitive DNA markers. $35 \mathrm{~S}$ rDNA - yellow; tandem repeats: GRS - pink, NTRS - orange, GRD3 - red, GRD5 - red with yellow centre, HRS60 blue and white (found only in N. sylvestris). Fluorescence in situ hybridisation of 35S rDNA on Nicotiana chromosomes (yellow signals) with chromosomes counterstained blue with DAPI. Note, dispersed yellow signals, particularly on the fourth pair of N. kawakamii chromosomes. Data and interpretative diagrams are taken from [17]. 


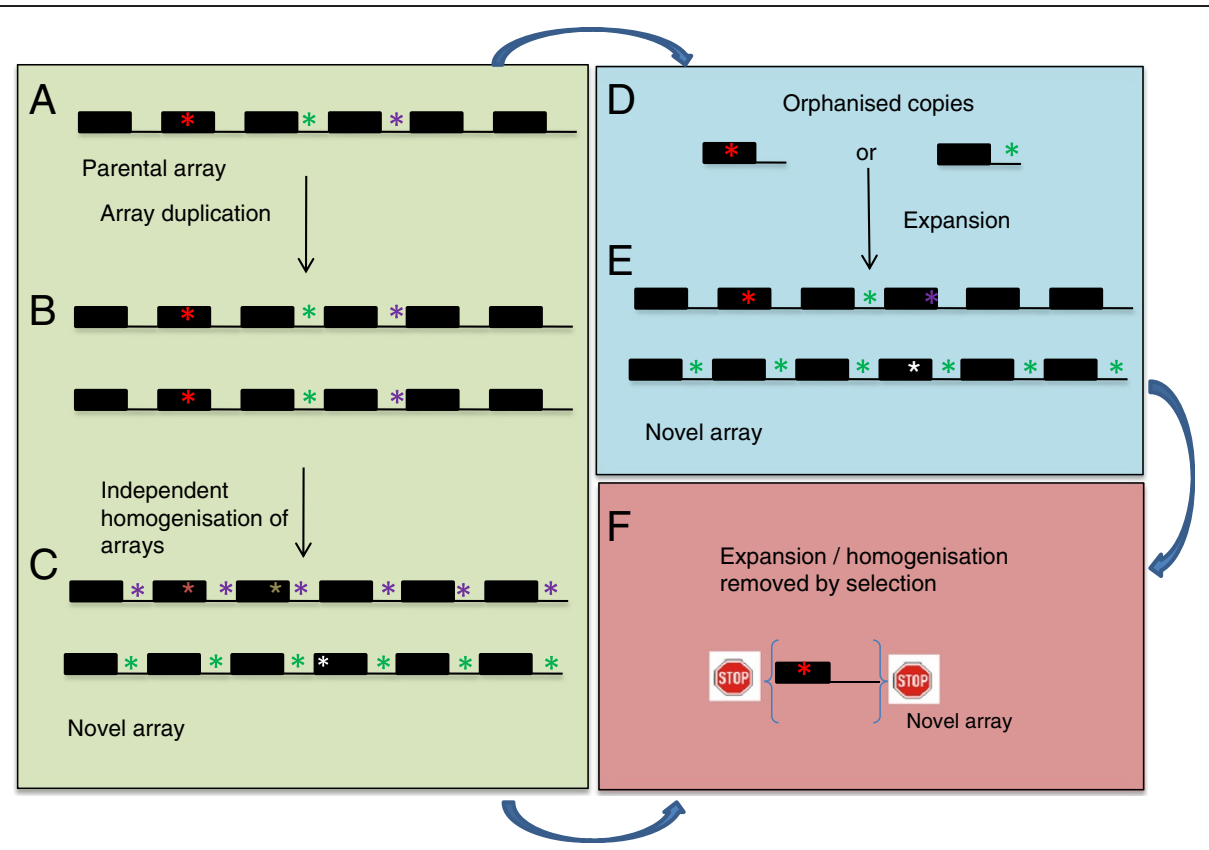

Figure 7 A theoretical model to explain rDNA divergence. Ribosomal DNA arrays (black box) separated by spacer sequences (black line) with some units carrying mutations (asterisks). Green panel: (A) $35 \mathrm{~S}$ rDNA locus comprising rDNA arrays with a few mutations. (B) Duplication of the $35 \mathrm{~S}$ rDNA locus, followed by (C) more rapid intrachromosomal homogenisation than interchromosomal homogenisation, leading to genetic divergence of both arrays and an increase in rDNA complexity. Mechanism would involve unequal recombination and gene conversion [2,69]. Blue panel: (D) Orphaned 35S rDNA copies may (E) nucleate locus expansion, resulting in long arrays of homogenous sequences. Pink panel: (F) Amplification of non-functional units will be selected against and lost from the population partially corroborating birth-and-death model of rDNA evolution [7,8].

loci $[62,63]$. A comparable pattern is reported within the grasshopper species Podisma pedestris, in which some populations have a larger number of rDNA loci and a greater complexity of rDNA units [64]. A similar correlation between locus number (Figure 6) and ITS1 complexity (Figure 2) emerges from our results. For example, $N$. tomentosiformis, with a single locus, has highly homogeneous $18 \mathrm{~S}$ and ITS1 sequences, resembling the situation in unicellular yeast. On the other hand, N. kawakamii with four loci had the highest variability amongst ITS sequences.

Why then is there a generally greater number of ribotype variants (Table 1) in species with more rDNA loci? The relationship could be explained if the rates of intrachromosomal homogenisation exceed those of interchromosomal homogenisation as has been proposed to explain the divergence of rDNA [65], satellite DNA [66] and retroelements [67]. The dynamics of homogenisation are analogous to the effects of gene flow: just as reduced gene flow allows variation to accumulate between geographically-separated populations, low levels of homogenisation between different chromosomal loci would allow the accumulation of different ribotypes (see Figure 7B-C). Another explanation is analogous to the effects of founder events in classical population genetics: if new rDNA loci are established by the movement or copying of a small number of units to a new location followed by an expansion in the number of these units (Figure 7D-E), then that too would result in higher inter-chromosomal variation. The patterns would also be shaped by selection: amplified rDNA variants containing deleterious mutations would be selected against (Figure 7E-F), partially explaining why we did not observe variation in the coding region (Figure 2). In contrast, functional arrays may rapidly expand to saturate the rDNA population, reducing selection pressures on older, more degenerate arrays. Certainly rDNA units incapacitated by transposon insertions amplify to considerable extent in Drosophila [68], suggesting differences in the levels to which individual genomes tolerate nonfunctional copies.

The rDNA variants could have arisen either through the amplification of low copy number units (Figure 7D-E), or through locus duplication (Figure 7A-C). Although the latter possibility seems to be a more likely there appears to be sufficient evolutionary time for homogenisation of a large number of units from a single orphaned copy (Figure 7D): theoretical computer simulations imply that the time taken for sequences arranged in tandem to homogenise is a quadratic function of their copy number [5]. It follows that a typical array comprising about 1000 units would homogenise within $\sim 10^{6}$ years. The age of Nicotiana diploid species $\left(\sim 10^{7}\right.$ years, [27]) exceeds that limit; the period since the speciation split of Nicotiana would therefore 
appear sufficiently long for the replacement of thousands of rDNA units in the genomes, which would explain why we do not detect common ribotypes shared between species (inherited from the progenitors), even in low frequency clusters. There is some indirect experimental support for the hypothesis that new loci may arise by the amplification of orphanised or low copy number rDNA sequences:

1. Low copy numbers of rDNA units (or parts of the unit) can be found dispersed across the Arabidopsis [70] and some animal [71,72] genomes. IGS dispersion may not be uncommon in plant genomes since it has also been reported in other species, and proposed to be one of the mechanisms leading to formation of novel high copy satellites [73-75]. Indeed, we have observed this phenomenon in Nicotiana section Tomentosae, where there is dispersion of IGS sequences and 35S rDNA across the genome [30]. Such units could potentially nucleate the expansion of active rDNA loci. Interestingly, $N$. kawakamii, with the highest level of rDNA dispersion (Figure 6), also has the most heterogeneous ITS1 ribotypes.

2. New loci and units have been shown to evolve rapidly in early generations of synthetic allopolyploids [31,7679] or in response to DNA damage [80]. In an allotetraploid line of tobacco, we observed amplification of new IGS types at a new rDNA locus [30].

3. Transposable elements may be vehicles for rDNA mobility and the generation of orphaned rDNA units across the genome. For example in wheat mobile rDNA sites may be connected with meiotic activity of En/Spm elements [81], while Ty1/Copia elements have been found in the IGS of Allium cernuum [82].

\section{Conclusions}

The results here indicate that ITS1 is more heterogeneous than $18 \mathrm{~S}$, particularly when more than one rDNA locus is present. Low homogenisation frequency between rDNA arrays at different loci, together with strong selection pressures imposed on coding regions, would allow co-existence of repeats with identical coding but more variable non-coding regions. We suggest that locus duplication or amplification of orphaned rDNA units can be vehicles for rDNA divergence (Figure 7).

\section{Additional files}

Additional file 1: List of $18 \mathrm{~S}$ gene clusters from $\mathbf{4 5 4}$ amplicon sequencing. Description: Each MsExcell list contains information about cluster ID, mutation pattern, number of reads, number of reads expressed as a percentage and total number of reads for a given species. For $N$. sylvestris and $N$. tomentosiformis, positions of variable sites read the noncoding DNA strands; for N. otophora and N. kawakamii, these read the coding strands.

Additional file 2: List of ITS1 clusters from 454 amplicon sequencing. The MsExcell charts are organized as in Additional file 1. Additional file 3: Substitution mutation patterns in ITS1 region (single read clusters were excluded).

Additional file 4: Abundance of individual clusters (number of reads/total number of reads per cluster, expressed as a percentage) in 454 sequencing data from $N$. tomentosiformis, $N$. sylvestris, $N$. otophora and $\boldsymbol{N}$. kawakamii ITS1 and $18 \mathrm{~S}$ rDNA sequences. The clusters are distinguished by polymorphisms, i.e. SNPs or indels.

Additional file 5: Output of SNP algorithm in CLC genomics of Illumina ITS1 reads from $N$. sylvestris and $N$. tomentosiformis. Additional file 6: Output of DIP algorithm in CLC genomics of Illumina ITS1 reads from $N$. sylvestris and $N$. tomentosiformis.

Additional file 7: Comparison of polymorphisms analyzed by different sequencing methods. The data are for N. sylvestris ITS1. Position (coding DNA strand) and type of mutation is in brackets: $[-]$ - no mutation (usually the most abundant cluster), $[57: C>T]-$ substitution $C$ into $T ;$ [ $49: 50>G]$ - insertion of $\mathrm{G}$ between nucleotides 49 and 50.

Additional file 8: Representation of IGS sequences in $\mathbf{4 5 4}$ reads. The plots show number of similarity hits along (A) N. sylvestris and (B) N. tomentosiformis, obtained in BLASTN searches against 454 reads from $\mathrm{N}$. sylvestris and N. tomentosiformis, respectively [22]. The analysis was run using the PROFREP server (http://w3lamc.umbr.cas.cz/profrep/public/) with e-value cutoff of 1e-15. The curves were smoothed by value averaging in a 10-bp sliding window. Conversion of the hit numbers to genomic copy numbers (per 1C) based on genome coverage of the 454 sequencing is provided on the right side of the plots.

\section{Competing interests}

The authors declare that they have no competing interests.

\section{Authors' contributions}

AK, ARL and RN designed the study. AK, ARL, SRB and RN wrote the paper. $R M, J F$ and MAG carried out most of the molecular biology part of the work. SRB, JM and RN carried out bioinformatic studies. All authors read and approved the final manuscript.

\section{Acknowledgements}

We thank Jana Kubičková for help with sorting the SNP analysis data. The work was supported by the Czech Science Foundation (P501/10/0208; P501/ 12/G090; 206/09/1751), Academy of Science of the Czech Republic (RVO68081707), the Czech-French bilateral Barrande program (09150SH), Natural Environment Research Council, UK, and Agence Nationale de la Recherche (ANR) research contract (Association pour la Recherche sur les Nicotianées).

\section{Author details}

'Institute of Biophysics, Academy of Sciences of the Czech Republic, v.v.i, Královopolská 135, Brno CZ-612 65, Czech Republic. ${ }^{2}$ Queen Mary University of London, School of Biological and Chemical Sciences, Mile End Road, London E1 4NS, UK. ${ }^{3}$ Department of Ecology, Evolution and Organismal Biology, lowa State University, Ames IA 50011, USA. ${ }^{4}$ Biology Centre, Academy of Sciences of the Czech Republic, Institute of Plant Molecular Biology, Branišovská 31, České Budějovice CZ-370 05, Czech Republic. ${ }^{5}$ Institut Jean-Pierre Bourgin, Laboratoire de Biologie Cellulaire, INRA-Centre de Versailles, Versailles Cedex F-780 26, France.

Received: 24 September 2012 Accepted: 13 December 2012 Published: 23 December 2012

\section{References}

1. Hemleben V, Zentgraf U: Structural organisation and regulation of transcription by RNA polymerase I of plant nuclear ribosomal genes. In Results and problems in cell differentiation 20: Plant promoters and 
transcription factors. Edited by N. L. Berlin/Heidelberg: Springer-Verlag; 1994:3-24

2. Dover GA: Molecular drive: a cohesive mode of species evolution. Nature 1982, 299:111-117.

3. Eickbush TH, Eickbush DG: Finely orchestrated movements: evolution of the ribosomal RNA genes. Genetics 2007, 175(2):477-485.

4. Feliner GN, Rossello JA: Concerted evolution of multigene families and homeologous recombination. In Plant Genome Diversity, Volume 1. Edited by Wendel JF. Wien: Springer-Verlag; 2012.

5. Smith GP: Unequal crossover and the evolution of multigene families. Cold Spring Harb Symp Quant Biol 1974, 38:507-513.

6. Ohta T: The mutational load of a multigene family with uniform members. Genet Research 1989, 53(2):141-145.

7. Rooney AP, Ward TJ: Evolution of a large ribosomal RNA multigene family in filamentous fungi: birth and death of a concerted evolution paradigm. Proc Natl Acad Sci USA 2005, 102(14):5084-5089.

8. Nei M, Rooney AP: Concerted and birth-and-death evolution of multigene families. Ann Rev Genet 2005, 39:121-152.

9. Harpke D, Peterson A: Non-concerted ITS evolution in Mammillaria (Cactaceae). Mol Phylogenet Evol 2006, 41(3):579-593.

10. Fehrer J, Krak K, Chrtek J Jr: Intra-individual polymorphism in diploid and apomictic polyploid hawkweeds (Hieracium, Lactuceae, Asteraceae): disentangling phylogenetic signal, reticulation, and noise. BmC Evol Biol 2009, 9:239.

11. Razafimandimbison SG, Kellogg EA, Bremer B: Recent origin and phylogenetic utility of divergent ITS putative pseudogenes: a case study from Naucleeae (Rubiaceae). Syst Biol 2004, 53(2):177-192.

12. Ingle J, Timmis JN, Sinclair J: The Relationship between Satellite Deoxyribonucleic Acid, Ribosomal Ribonucleic Acid Gene Redundancy, and Genome Size in Plants. Plant Physiol 1975, 55(3):496-501.

13. Heslop-Harrison JS, Schwarzacher T: Organisation of the plant genome in chromosomes. Plant J 2011, 66(1):18-33.

14. Song JY, Shi LC, Li DZ, Sun YZ, Niu YY, Chen ZD, Luo HM, Pang XH, Sun ZY, Liu C, et al: Extensive Pyrosequencing Reveals Frequent Intra-Genomic Variations of Internal Transcribed Spacer Regions of Nuclear Ribosomal DNA. Plos One 2012, 7(8):43971.

15. Straub SC, Parks M, Weitemier K, Fishbein M, Cronn RC, Liston A: Navigating the tip of the genomic iceberg: Next-generation sequencing for plant systematics. Am J Bot 2012, 99(2):349-364.

16. Kenton A, Parokonny AS, Gleba YY, Bennett MD: Characterization of the Nicotiana tabacum L. genome by molecular cytogenetics. Mol Gen Genet 1993, 240(2):159-169.

17. Lim KY, Matyasek R, Lichtenstein CP, Leitch AR: Molecular cytogenetic analyses and phylogenetic studies in the Nicotiana section Tomentosae. Chromosoma 2000, 109(4):245-258.

18. Leitch AR, Lim KY, Kovarik A, Matyasek R, Chase MW, Knapp S, McCarthy E, Clarkson JJ: Comparative genomics and repetitive sequence divergence in the species of diploid Nicotiana section Alatae. Plant J 2006, 48(6):907-919.

19. Koukalova B, Moraes AP, Renny-Byfield S, Matyasek R, Leitch AR, Kovarik A: Fall and rise of satellite repeats in allopolyploids of Nicotiana over c. 5 million years. New Phytol 2010, 186(1):148-160.

20. Matyasek R, Fulnecek J, Leitch AR, Kovarik A: Analysis of two abundant, highly related satellites in the allotetraploid Nicotiana arentsii using double-strand conformation polymorphism analysis and sequencing. New Phytol 2011, 192(3):747-759.

21. Ren N, Timko MP: AFLP analysis of genetic polymorphism and evolutionary relationships among cultivated and wild Nicotiana species. Genome 2001, 44(4):559-571.

22. Renny-Byfield S, Chester M, Kovařík A, LC SC, Grandbastien MA MD Nichols R, Macas J, Novák P, Leitch AR: Next generation sequencing reveals evidence of genome downsizing and elimination of paternally derived repetitive DNA sequences in tobacco. Mol Biol Evol 2011, 28:2843-2854

23. Renny-Byfield S, Kovarik A, Chester M, Nichols RA, Macas J, Novak P, Leitch AR: Independent, Rapid and Targeted Loss of Highly Repetitive DNA in Natural and Synthetic Allopolyploids of Nicotiana tabacum. Plos One 2012, 7(5):e36963.

24. Bogani $\mathrm{P}$, Lio $\mathrm{P}$, Intrieri MC, Buiatti M: A physiological and molecular analysis of the genus Nicotiana. Mol Phylogenet Evol 1997, 7(1):62-70.

25. Clarkson JJ, Kelly LJ, Leitch AR, Knapp S, Chase MW: Nuclear glutamine synthetase evolution in Nicotiana: Phylogenetics and the origins of allotetraploid and homoploid (diploid) hybrids. Mol Phylogenet Evol 2010, 55(1):99-112.

26. Aoki S, Ito M: Molecular phylogeny of Nicotiana (Solanaceae) based on the nucleotide sequence of the matK gene. Plant Biology 2000, 2:316-324.

27. Chase MW, Knapp S, Cox AV, Clarkson JJ, Butsko Y, Joseph J, Savolainen V, Parokonny AS: Molecular systematics, GISH and the origin of hybrid taxa in Nicotiana (Solanaceae). Ann Bot-London 2003, 92(1):107-127.

28. Moscone EA, Matzke MA, Matzke AJ: The use of combined FISH/GISH in conjunction with DAPI counterstaining to identify chromosomes containing transgene inserts in amphidiploid tobacco. Chromosoma 1996, 105(5):231-236.

29. Lim KY, Kovarik A, Matyasek R, Chase MW, Knapp S, McCarthy E, Clarkson JJ, Leitch AR: Comparative genomics and repetitive sequence divergence in the species of diploid Nicotiana section Alatae. Plant J 2006 48(6):907-919

30. Lim KY, Skalicka K, Koukalova B, Volkov RA, Matyasek R, Hemleben V, Leitch $A R$, Kovarik A: Dynamic changes in the distribution of a satellite homologous to intergenic 26-18S rDNA spacer in the evolution of Nicotiana. Genetics 2004, 166(4):1935-1946.

31. Skalicka K, Lim KY, Matyasek R, Koukalova B, Leitch AR, Kovarik A: Rapid evolution of parental rDNA in a synthetic tobacco allotetraploid line. Am J Bot 2003, 90(7):988-996.

32. Goodspeed TH: The genus Nicotiana, Volume 16. Massachusetts, USA: Waltham; 1954.

33. Lim KY, Matyasek R, Kovarik A, Leitch A: Genome evolution in allotetraploid Nicotiana. Biol J Linn Soc 2004, 82(4):599-606.

34. Volkov RA, Borisjuk NV, Panchuk II, Schweizer D, Hemleben V: Elimination and rearrangement of parental rDNA in the allotetraploid Nicotiana tabacum. Mol Biol Evol 1999, 16(3):311-320.

35. Kovarik A, Matyasek R, Lim KY, Skalicka K, Koukalova B, Knapp S, Chase M, Leitch AR: Concerted evolution of 18-5.8-26S rDNA repeats in Nicotiana allotetraploids. Biol J Linn Soc 2004, 82(4):615-625.

36. Williams R, Peisajovich SG, Miller OJ, Magdassi S, Tawfik DS, Griffiths AD: Amplification of complex gene libraries by emulsion PCR. Nat Methods 2006, 3(7):545-550

37. Kovarik A, Pires JC, Leitch AR, Lim KY, Sherwood AM, Matyasek R, Rocca J, Soltis DE, Soltis PS: Rapid concerted evolution of nuclear ribosomal DNA in two Tragopogon allopolyploids of recent and recurrent origin. Genetics 2005, 169(2):931-944

38. Hall TA: BioEdit: a user-friendly biological sequence alignment editor and analysis program for Windows 95/98/NT. Nucl Acids Symp Ser 1999, 41:95-98.

39. Buckler ES, Ippolito A, Holtsford TP: The evolution of ribosomal DNA: divergent paralogues and phylogenetic implications. Genetics 1997, 145(3):821-832.

40. Keller I, Chintauan-Marquier IC, Veltsos P, Nichols RA: Ribosomal DNA in the grasshopper Podisma pedestris: escape from concerted evolution. Genetics 2006, 174(2):863-874

41. Kovarik A, Koukalova B, Lim KY, Matyasek R, Lichtenstein CP, Leitch AR, Bezdek M: Comparative analysis of DNA methylation in tobacco heterochromatic sequences. Chromosome Res 2000, 8(6):527-541.

42. Garcia S, Garnatje T, Kovarik A: Plant rDNA database: ribosomal DNA loci data including other karyological and cytogenetic information in plants. Chromosoma 2012, 121(4):389-394.

43. Lim KY, Kovarik A, Matyasek R, Bezdek M, Lichtenstein CP, Leitch AR: Gene conversion of ribosomal DNA in Nicotiana tabacum is associated with undermethylated, decondensed and probably active gene units. Chromosoma 2000, 109(3):161-172.

44. Miller JR, Delcher AL, Koren S, Venter E, Walenz BP, Brownley A, Johnson J, Li K, Mobarry C, Sutton G: Aggressive assembly of pyrosequencing reads with mates. Bioinformatics 2008, 24(24):2818-2824.

45. Knapp S, Chase MW, Clarkson JJ: Nomenclatural changes and a new sectional classification in Nicotiana (Solanaceae). Taxon 2004, 53(1):73-82

46. Ganley AR, Kobayashi T: Highly efficient concerted evolution in the ribosomal DNA repeats: total rDNA repeat variation revealed by wholegenome shotgun sequence data. Genome Res 2007, 17(2):184-191.

47. Stage DE, Eickbush TH: Sequence variation within the rRNA gene loci of 12 Drosophila species. Genome Res 2007, 17(12):1888-1897.

48. Carvalho A, Guedes-Pinto H, Lima-Brito J: Intergenic spacer length variants in Old Portuguese bread wheat cultivars. J Genet 2011, 90 (2):203-208 
49. Saghai-Maroof MA, Soliman KM, Jorgensen RA, Allard RW: Ribosomal DNA spacer-length polymorphisms in barley: mendelian inheritance, chromosomal location, and population dynamics. Proc Natl Acad Sci USA 1984, 81(24):8014-8018.

50. Volkov R, Kostishin S, Ehrendorfer E, Schweizer D: Molecular organization and evolution of the external transcribed rDNA spacer region in two diploid relatives of Nicotiana tabacum (Solanaceae). Plant Syst Evol 1996, 201(1-4):117-129.

51. Ambrose CD, Crease TJ: Evolution of the nuclear ribosomal DNA intergenic spacer in four species of the Daphnia pulex complex. BMC Genet 2011, 12:13. doi:10.1186/1471-2156-12-13.

52. Dvorak J, Jue D, Lassner M: Homogenization of Tandemly Repeated Nucleotide-Sequences by Distance-Dependent Nucleotide-Sequence Conversion. Genetics 1987, 116(3):487-498.

53. Kitamura S, Inoue M, Shikazono N, Tanaka A: Relationships among Nicotiana species revealed by the 5S rDNA spacer sequence and fluorescence in situ hybridization. Theor App/ Genet 2001, 103(5): 678-686.

54. Clarkson JJ, Knapp S, Garcia VF, Olmstead RG, Leitch AR, Chase MW: Phylogenetic relationships in Nicotiana (Solanaceae) inferred from multiple plastid DNA regions. Mol Phylogenet Evol 2004, 33(1):75-90.

55. Murad L, Lim KY, Christopodulou V, Matyasek R, Lichtenstein C, Kovarik A Leitch A: The origin of tobacco's T genome is tracted to a particular lineage within Nicotiana tomentosiformis (Solanaceae). Am J Bot 2002, 89(6):921-928.

56. Maddison WP: Gene trees in species trees. Syst Biol 1997, 46(3):523-536

57. Degnan $\mathrm{JH}$, Rosenberg NA: Gene tree discordance, phylogenetic inference and the multispecies coalescent. Trends Ecol Evol 2009, 24 (6):332-340

58. Blattner FR: Phylogenetic analysis of Hordeum (Poaceae) as inferred by nuclear rDNA ITS sequences. Mol Phylogenet Evol 2004, 33(2):289-299.

59. Simon UK, Trajanoski S, Kroneis T, Sedlmayr P, Guelly C, Guttenberger H: Accession-Specific Haplotypes of the Internal Transcribed Spacer Region in Arabidopsis thaliana-A Means for Barcoding Populations. Mol Biol Evol 2012, 29(9):2231-2239.

60. Ritz CM, Schmuths H, Wissemann V: Evolution by reticulation: European dogroses originated by multiple hybridization across the genus Rosa. $J$ Hered 2005, 96(1):4-14

61. Kovarik A, Werlemark G, Leitch AR, Souckova-Skalicka K, Lim YK, Khaitova L, Koukalova B, Nybom H: The asymmetric meiosis in pentaploid dogroses (Rosa sect. Caninae) is associated with a skewed distribution of rRNA gene families in the gametes. Heredity (Edinb) 2008, 101(4):359-367.

62. Penas MLL, Urdampilleta JD, Bernardello G, Forni-Martins ER: Karyotypes, heterochromatin, and physical mapping of 18S-26S rDNA in Cactaceae. Cytogenet Genome Res 2009, 124(1):72-80.

63. Lim KY, Werlemark G, Matyasek R, Bringloe JB, Sieber V, El Mokadem H, Meynet J, Hemming J, Leitch AR, Roberts AV: Evolutionary implications of permanent odd polyploidy in the stable sexual, pentaploid of Rosa canina L. Heredity (Edinb) 2005, 94(5):501-506.

64. Veltsos P, Keller I, Nichols RA: Geographically localised bursts of ribosomal DNA mobility in the grasshopper Podisma pedestris. Heredity (Edinb) 2009, 103(1):54-61.

65. Schlotterer C, Tautz D: Chromosomal homogeneity of Drosophila ribosomal DNA arrays suggests intrachromosomal exchanges drive concerted evolution. Current Biol 1994, 4(9):777-783.

66. Krzywinski J, Sangare D, Besansky NJ: Satellite DNA from the $Y$ chromosome of the malaria vector Anopheles gambiae. Genetics 2005, 169(1):185-196.

67. Kejnovsky E, Hobza R, Kubat Z, Widmer A, Marais GAB, Vyskot B: High intrachromosomal similarity of retrotransposon long terminal repeats: Evidence for homogenization by gene conversion on plant sex chromosomes? Gene 2007, 390(1-2):92-97.

68. Eickbush TH, Burke WD, Eickbush DG, Lathe WC: Evolution of R1 and R2 in the rDNA units of the genus Drosophila. Genetica 1997, 100(1-3):49-61.

69. Brown DD, Wensink PC, Jordan E: Comparison of Ribosomal Dnas of Xenopus-Laevis and Xenopus-Mulleri - Evolution of Tandem Genes. J Mol Biol 1972, 63(1):57-73.

70. Mentewab AB, Jacobsen MJ, Flowers RA: Incomplete homogenization of $18 \mathrm{~S}$ ribosomal DNA coding regions in Arabidopsis thaliana. BMC Res Notes 2011, 4:93.
71. Guimond A, Moss T: A ribosomal orphon sequence from Xenopus laevis flanked by novel low copy number repetitive elements. Biol Chem 1999, 380(2):167-174.

72. Munro J, Burdon RH, Leader DP: Characterization of a Human Orphon 28 S Ribosomal DNA. Gene 1986, 48(1):65-70.

73. Almeida C, Fonseca A, dos Santos KGB, Mosiolek M, Pedrosa-Harand A Contrasting evolution of a satellite DNA and its ancestral IGS rDNA in Phaseolus (Fabaceae). Genome 2012, 55(9):683-689.

74. Jo SH, Park HM, Kim SM, Kim HH, Hur CG, Choi D: Unraveling the sequence dynamics of the formation of genus-specific satellite DNAs in the family solanaceae. Heredity (Edinb) 2011, 106(5):876-885.

75. Macas J, Navratilova A, Meszaros T: Sequence subfamilies of satellite repeats related to rDNA intergenic spacer are differentially amplified on Vicia sativa chromosomes. Chromosoma 2003, 112(3):152-158.

76. Pontes O, Neves N, Silva M, Lewis MS, Madlung A, Comai L, Viegas W, Pikaard CS: Chromosomal locus rearrangements are a rapid response to formation of the allotetraploid Arabidopsis suecica genome. Proc Natl Acad Sci USA 2004, 101(52):18240-18245.

77. Xiong ZY, Gaeta RT, Pires JC: Homoeologous shuffling and chromosome compensation maintain genome balance in resynthesized allopolyploid Brassica napus. Proc Natl Acad Sci USA 2011, 108(19):7908-7913.

78. Ksiazczyk T, Kovarik A, Eber F, Huteau V, Khaitova L, Tesarikova Z, Coriton O, Chevre AM: Immediate unidirectional epigenetic reprogramming of NORs occurs independently of rDNA rearrangements in synthetic and natural forms of a polyploid species Brassica napus. Chromosoma 2011, 120(6):557-571.

79. Shcherban AB, Badaeva ED, Amosova AV, Adonina IG, Salina EA: Genetic and epigenetic changes of rDNA in a synthetic allotetraploid. Aegilops sharonensis $x$ Ae. umbellulata. Genome 2008, 51(4):261-271.

80. Fukuoka H, Kageyama Y, Yamamoto K, Takeda G: Rapid conversion of rDNA intergenic spacer of diploid mutants of rice derived from gammaray irradiated tetraploids. Mol Gen Genet 1994, 243(2):166-172.

81. Raskina O, Belyayev A, Nevo E: Activity of the En/Spm-like transposons in meiosis as a base for chromosome repatterning in a small, isolated, peripheral population of Aegilops speltoides Tausch. Chromosome Res 2004, 12(2):153-161.

82. Chester M, Sykorova E, Fajkus J, Leitch AR: Single integration and spread of a copia-like sequence nested in rDNA intergenic spacers of Allium cernuum (Alliaceae). Cytogenet Genome Res 2010, 129(1-3):35-46.

doi:10.1186/1471-2164-13-722

Cite this article as: Matyášek et al:: Next generation sequencing analysis reveals a relationship between rDNA unit diversity and locus number in Nicotiana diploids. BMC Genomics 2012 13:722.

\section{Submit your next manuscript to BioMed Central and take full advantage of:}

- Convenient online submission

- Thorough peer review

- No space constraints or color figure charges

- Immediate publication on acceptance

- Inclusion in PubMed, CAS, Scopus and Google Scholar

- Research which is freely available for redistribution 